\title{
Generalized Reliability Bounds For Coherent Structures
}

\author{
Michael V. Boutsikas* and Markos V. Koutras \\ Department of Mathematics, University of Athens, Greece 15784
}

\begin{abstract}
In this article we introduce generalizations of several well known reliability bounds. These bounds are based on arbitrary partitions of the family of minimal path or cut sets of the system and can be used for approximating the reliability of any coherent structure with iid components. An illustration is also given of how the general results can be applied for a specific reliability structure (two-dimensional consecutive- $k_{1} \times k_{2^{-}}$ out-of- $n_{1} \times n_{2}$ system) along with extensive numerical calculations revealing that, in most cases, the generalized bounds perform better than other available bounds in the literature for this system.
\end{abstract}

Keywords: Reliability bounds; cut sets; path sets; association; binary coherent structures; two-dimensional consecutive- $k_{1} \times k_{2}$-out-of- $n_{1} \times n_{2}$ system.

AMS 1991 Subject Classifications: Primary 60E15, Secondary 60K10

\section{Introduction}

Let us consider a coherent reliability structure with $n$ independent components and denote by $I$ the finite set of all of its components and by

$$
\mathbb{C}=\left\{C_{1}, C_{2}, \ldots, C_{N}\right\}, \quad \mathbb{P}=\left\{P_{1}, P_{2}, \ldots, P_{M}\right\}
$$

the family of system's minimal cut and path sets respectively. Let us also denote by $X_{i}, i \in I$ the state of component $i$ at a fixed instance (i.e. $X_{i}=0$ if the component is failed and $X_{i}=1$ if the component functions) and by $p_{i}=E\left(X_{i}\right), q_{i}=1-p_{i}$, the respective survival and failure probabilities. Despite the existence of compact formulae for the structure function of the system (c.f. Barlow and Proschan (1981)), namely

$$
\phi(\mathbf{X})=\prod_{C \in \mathbb{C}}\left(1-\prod_{i \in C}\left(1-X_{i}\right)\right)=1-\prod_{P \in \mathbb{P}}\left(1-\prod_{i \in P} X_{i}\right)
$$

\footnotetext{
${ }^{*}$ Research supported by the National Scholarship Foundation of Greece.
} 
the computation of the reliability $R=E(\phi)$ is in general a laborious task to perform. This is mainly due to the fact that the reduction of $\phi$ in a sum of products of $X_{i}$ usually becomes unwieldy, especially in the situations where there exists a large number of minimal cut or path sets. It is, therefore, not surprising that special attention has been drawn to the development of efficient reliability approximation formulae which do not require a sum-ofproducts decomposition.

One of the earliest approaches to this problem should be attributed to Esary and Proschan (1963) who derived the simple reliability bounds

$$
L_{E P}=\prod_{C \in \mathbb{C}}\left(1-\prod_{i \in C}\left(1-p_{i}\right)\right) \leq R \leq 1-\prod_{P \in \mathbb{P}}\left(1-\prod_{i \in P} p_{i}\right)=U_{E P}
$$

(see also Barlow and Proschan (1981)). Recently, Fu and Koutras (1995) established some new reliability bounds that can be efficiently combined with $L_{E P}, U_{E P}$ to construct tight approximating intervals for $R$. More specifically they proved that

$$
L_{F K}=1-\prod_{j=1}^{M}\left(1-\prod_{i \in K_{j}} q_{i} \prod_{i \in P_{j}} p_{i}\right) \leq R \leq \prod_{j=1}^{N}\left(1-\prod_{i \in L_{j}} p_{i} \prod_{i \in C_{j}} q_{i}\right)=U_{F K}
$$

where $L_{j}, K_{j}$ are appropriate index sets $\left(L_{j}, K_{j} \subset I\right)$ determined by a careful examination of the minimal cut and path set families $\mathbb{C}, \mathbb{P}$.

Exploiting a general covariance bound established recently by Boutsikas and Koutras (1999) one can easily arrive at the following bounds which use the upper Esary and Proschan bound to arrive at a lower reliability bound $L_{C B}$ and the lower Esary and Proschan bound to arrive at an upper reliability bound $U_{C B}$ :

$$
L_{C B}=U_{E P}-\sum_{b<a}\left(\prod_{i \in P_{a} \cup P_{b}} p_{i}-\prod_{i \in P_{a}} p_{i} \prod_{i \in P_{b}} p_{i}\right), U_{C B}=L_{E P}+\sum_{b<a}\left(\prod_{i \in C_{a} \cup C_{b}} q_{i}-\prod_{i \in C_{a}} q_{i} \prod_{i \in C_{b}} q_{i}\right) .
$$

Finally we mention the classical min-max bounds (see e.g. Barlow and Proschan (1981))

$$
L_{M}=\max _{1 \leq j \leq M} \prod_{i \in P_{j}} p_{i} \leq R \leq \min _{1 \leq j \leq N}\left(1-\prod_{i \in C_{j}}\left(1-p_{i}\right)\right)=U_{M}
$$

which offer usually crude but easy to evaluate reliability approximations.

In the present article we generalize all the aforementioned bounds by considering an arbitrary partition of the family of minimal cut and path sets. The organization of the paper is as follows. In Section 2 we introduce some preliminary definitions and notations that will be extensively used throughout our exposition. In Section 3 we present classes of bounds which are constructed on arbitrary partitions of the families of minimal cut or path sets and include as special cases the reliability bounds (2)-(5). Our bounds are not expressed in explicit form in terms of component reliabilities as bounds (2)-(5) are. They involve the reliabilities of certain subsystems of reduced size and complexity (as compared to the system 
under investigation); therefore, they can be profitably brought into play once there are available simple formulae or algorithms for the evaluation of the subsystems' reliabilities. In Section 4 we indicate how the general results of Section 3 can be applied to a specific reliability structure, namely the two-dimensional consecutive- $k_{1} \times k_{2}$-out-of- $n_{1} \times n_{2}$ system. As the extensive numerical experimentation conducted in this section revealed, the new bounds perform, in most cases, better than the bounds having appeared in the literature so far. Further applications of the general results to other interesting reliability structures such as $r$-within-consecutive- $k$-out-of- $n$ system, and $r$-within-two-dimensional consecutive$k_{1} \times k_{2}$-out-of- $n_{1} \times n_{2}$ system will be presented in a subsequent article.

\section{Definitions and notations}

The theory of associated random variables (r.v.'s) will play a crucial role in the establishment of the main results of the present work. A collection of r.v.'s $Y_{1}, Y_{2}, \ldots, Y_{m}$ is said to be associated if for every pair of coordinatewise non-decreasing functions $f$ and $g$,

$$
\operatorname{Cov}(f(\mathbf{Y}), g(\mathbf{Y})) \geq 0
$$

where $\mathbf{Y}=\left(Y_{1}, Y_{2}, \ldots, Y_{m}\right)$. Throughout we assume, without further explicit mention, that the functions $f$ and $g$ are such that $\operatorname{Cov}(f(\mathbf{Y}), g(\mathbf{Y}))$ exists.

We summarize below several elementary properties of the class of associated r.v.'s which are referenced in the sequel.

- P1. Any subset of associated r.v.'s are associated.

- P2. If two sets of associated r.v.'s are independent of one another, then their union is a set of associated r.v.'s.

- P3. A single r.v. is associated.

- P4. Coordinatewise non-decreasing functions of associated r.v.'s are associated.

- P5. If $Y_{1}, \ldots, Y_{m}$ are binary associated r.v.'s then $1-Y_{1}, \ldots, 1-Y_{m}$ are also associated.

For the proof of P1-P5 and a more complete list, the reader might wish to consult Esary et al. (1967) or Barlow and Proschan (1981).

The following theorem, which was derived by Boutsikas and Koutras (1999), will also be used for the development of some general reliability bounds in the next paragraph.

Theorem 1 If $Y_{1}, Y_{2}, \ldots, Y_{m}$ are associated non-negative, integer valued r.v.'s, such that $E\left(Y_{i}\right), E\left(Y_{i} Y_{j}\right)<\infty, i, j=1,2, \ldots, m, i \neq j$, then

$$
0 \leq P\left(Y_{i}=0, i=1,2, \ldots, m\right)-\prod_{i=1}^{m} P\left(Y_{i}=0\right) \leq \sum_{i<j} \operatorname{Cov}\left(Y_{i}, Y_{j}\right)
$$


For the special case of binary associated r.v.'s, we get (applying the above inequality for $\left.1-Y_{i}\right)$

$$
0 \leq E\left(\prod_{i=1}^{m} Y_{i}\right)-\prod_{i=1}^{m} E\left(Y_{i}\right) \leq \sum_{i<j} \operatorname{Cov}\left(Y_{i}, Y_{j}\right)
$$

The LHS of (6) provides a lower bound for $E\left(\prod_{i=1}^{m} Y_{i}\right)$; this bound was first pointed out by Esary et al. (1967). On the other hand, the RHS indicates that the "total covariance" $\sum_{i<j} \operatorname{Cov}\left(Y_{i}, Y_{j}\right)$ can be used as an estimate of the maximum error incurred when $E\left(\prod_{i=1}^{m} Y_{i}\right)$ is approximated by $\prod_{i=1}^{m} E\left(Y_{i}\right)$.

If $X_{i}, i \in I$ are the state variables of the $n$ components of a coherent structure, then $Y_{j}=1-\prod_{i \in C_{j}}\left(1-X_{i}\right), j=1,2, \ldots, N$ are associated (by P2, P3 - which guarantee that a set of independent r.v.'s is associated - and P4). Likewise, $Y_{j}=1-\prod_{i \in P_{j}} X_{i}, j=1,2, \ldots, M$ are associated (by P2, P3, P4 and P5) and a direct application of (6) yields the reliability bounds $L_{C B}, U_{C B}$ mentioned in the introduction.

We shall now proceed to the introduction of some notations that will be used throughout the paper. If $I$ is the set of system's components and $\mathbb{A}$ a collection of subsets of $I$, we define

$$
\delta_{C}(\mathbb{A})=\prod_{A \in \mathbb{A}}\left(1-\prod_{i \in A}\left(1-X_{i}\right)\right), \quad \delta_{P}(\mathbb{A})=1-\prod_{A \in \mathbb{A}}\left(1-\prod_{i \in A} X_{i}\right)
$$

and $R_{C}(\mathbb{A})=E\left(\delta_{C}(\mathbb{A})\right), R_{P}(\mathbb{A})=E\left(\delta_{P}(\mathbb{A})\right)$ (Assumption: products and sums defined over empty sets will be set hereafter equal to 1 and 0 respectively; thus $\left.\delta_{C}(\emptyset)=1, \delta_{P}(\emptyset)=0\right)$. Manifestly, the reliability function of a system can be expressed as

$$
R=R(\mathbf{p})=R_{C}(\mathbb{C})=R_{P}(\mathbb{P}), \mathbf{p}=\left(p_{i}, i \in I\right)
$$

whereas the $E P$ bounds take on the form

$$
L_{E P}=\prod_{C \in \mathbb{C}} R_{C}(\{C\}), U_{E P}=1-\prod_{P \in \mathbb{P}} R_{P}(\{P\}) .
$$

From now on, the symbols $\mathcal{C}, \mathcal{P}$ will denote an arbitrary partition of $\mathbb{C}, \mathbb{P}$ respectively, i.e. $\mathcal{C}=\left\{\mathbb{C}_{1}, \mathbb{C}_{2}, \ldots, \mathbb{C}_{\nu}\right\}, \mathcal{P}=\left\{\mathbb{P}_{1}, \mathbb{P}_{2}, \ldots, \mathbb{P}_{\mu}\right\}$ where

- $\mathbb{C}_{s} \subset \mathbb{C}$ for $s=1,2, \ldots, \nu, \mathbb{C}_{i} \cap \mathbb{C}_{j}=\emptyset$ for $i \neq j$ and $\bigcup_{s=1}^{\nu} \mathbb{C}_{s}=\mathbb{C}$,

- $\mathbb{P}_{s} \subset \mathbb{P}$ for $s=1,2, \ldots, \mu, \mathbb{P}_{i} \cap \mathbb{P}_{j}=\emptyset$ for $i \neq j$ and $\bigcup_{s=1}^{\mu} \mathbb{P}_{s}=\mathbb{P}$.

\section{Generalized bounds}

It is clear that the lower bounds $L_{E P}$ (c.f. (2) and (7)) can be obtained by considering a series system with $|\mathbb{C}|$ independent subsystems, each subsystem being a parallel structure whose components are the ones contained in $C \in \mathbb{C}$ (this interpretation is valid on the 
understanding that each set of replicated components in the minimal cut sets is replaced by a corresponding set of independently operating components of the same reliability).

A natural question arising here is the following: can one combine several minimal cut sets together, therefore reducing the number of series subsystems and increasing subsystems' size? The next theorem answers this question to the positive. More specifically, it is shown that any partition of $\mathbb{C}$ gives birth to a lower reliability bound.

Needless to say, the same arguments can be easily extended (with some trivial modifications) for path sets as well, thereof obtaining upper reliability bounds.

Theorem 2 The reliability $R$ of any coherent structure is bounded below and above as follows

$$
\prod_{s=1}^{\nu} R_{C}\left(\mathbb{C}_{s}\right) \leq R \leq 1-\prod_{s=1}^{\mu}\left(1-R_{P}\left(\mathbb{C}_{s}\right)\right)
$$

Proof. The r.v.'s $\delta_{C}\left(\mathbb{C}_{s}\right), s=1,2, \ldots, \nu$ are coordinatewise non-decreasing functions of the independent (and hence associated, by P2, P3) r.v.'s $X_{i}, i \in I$. Therefore, by virtue of $\mathrm{P} 4$, they are associated and making use of the LHS inequality of (6) we deduce

$$
R=E\left(\prod_{C \in \mathbb{C}}\left(1-\prod_{i \in C}\left(1-X_{i}\right)\right)\right)=E\left(\prod_{s=1}^{\nu} \delta_{C}\left(\mathbb{C}_{s}\right)\right) \geq \prod_{s=1}^{\nu} E\left(\delta_{C}\left(\mathbb{C}_{s}\right)\right)=\prod_{s=1}^{\nu} R_{C}\left(\mathbb{C}_{s}\right) .
$$

Employing exactly the same arguments for the r.v's $1-\delta_{P}\left(\mathbb{P}_{s}\right), s=1,2, \ldots, \mu$, we may write

$$
\begin{aligned}
R & =E\left(1-\prod_{P \in \mathbb{P}}\left(1-\prod_{i \in P} X_{i}\right)\right)=1-E\left(\prod_{s=1}^{\mu}\left(1-\delta_{P}\left(\mathbb{P}_{s}\right)\right)\right) \\
& \left.\leq 1-\prod_{s=1}^{\mu} E\left(1-\delta_{P}\left(\mathbb{P}_{s}\right)\right)=1-\prod_{s=1}^{\mu}\left(1-R_{P}\left(\mathbb{P}_{s}\right)\right)\right)
\end{aligned}
$$

which concludes the proof.

It is worth mentioning that the proof of Theorem 2 carries over nicely to the case of having associated components $\left(X_{i}, i \in I\right.$ are associated r.v.'s) instead of independent ones. The former condition is quite realistic in many reliability analyses (see e.g. Barlow and Proschan (1981)). The reliability bounds put forth by Theorem 2 will be denoted by

$$
L_{E P}^{(G)}=\prod_{s=1}^{\nu} R_{C}\left(\mathbb{C}_{s}\right), U_{E P}^{(G)}=1-\prod_{s=1}^{\mu}\left(1-R_{P}\left(\mathbb{P}_{s}\right)\right)
$$

and will be referenced as generalized Esary and Proschan bounds. It is clear that, for the two extreme cases $\nu=N, \mathbb{C}_{s}=\left\{C_{s}\right\}, s=1,2, \ldots, \nu$ and $\nu=1, \mathbb{C}_{1}=\mathbb{C}$, the lower bounds $L_{E P}^{(G)}$ reduce to the usual $E P$ bound (1.2) and exact reliability $R$ respectively. It is not difficult to check that a gradual progress from the one extreme case $\nu=N$ to the other $\nu=1$ results in a monotone improvement of the generalized bounds; more precisely, if two partitions $\mathcal{C}, \mathcal{C}^{\prime}$ 
are available and for each $\mathbb{A} \in \mathcal{C}$ there exists an $\mathbb{A}^{\prime} \in \mathcal{C}^{\prime}$ such that $\mathbb{A} \subset \mathbb{A}^{\prime}$ then the $L_{E P}^{(G)}$ associated with $\mathcal{C}$ is worse (smaller) than the $L_{E P}^{(G)}$ associated with $\mathcal{C}^{\prime}$. Usually, the cost paid for the improvement of the bounds as the partitions' members become thicker, is the increasing computational complexity of the bounds themselves. It goes without saying that similar remarks can be easily stated (under some obvious adjustments) for minimal path sets and generalized upper bounds as well.

Let us next proceed to the establishment of partition-based bounds which generalize the covariance bounds (4).

Theorem 3 The reliability $R$ of any coherent structure is bounded below and above by

$$
\begin{aligned}
L_{C B}^{(G)} & =U_{E P}^{(G)}-\sum_{1 \leq b<a \leq \mu}\left(1-R_{P}\left(\mathbb{P}_{a} \cup \mathbb{P}_{b}\right)-\left(1-R_{P}\left(\mathbb{P}_{a}\right)\right)\left(1-R_{P}\left(\mathbb{P}_{b}\right)\right)\right) \\
& \geq U_{E P}^{(G)}-\sum_{1 \leq b<a \leq \mu} \sum_{P \in \mathbb{P}_{a}} \sum_{Q \in \mathbb{P}_{b}}\left(\prod_{i \in P \cup Q} p_{i}-\prod_{i \in P} p_{i} \prod_{i \in Q} p_{i}\right)
\end{aligned}
$$

and

$$
\begin{aligned}
U_{C B}^{(G)} & =L_{E P}^{(G)}+\sum_{1 \leq b<a \leq \nu}\left(R_{C}\left(\mathbb{C}_{a} \cup \mathbb{C}_{b}\right)-R_{C}\left(\mathbb{C}_{a}\right) R_{C}\left(\mathbb{C}_{b}\right)\right) \\
& \leq L_{E P}^{(G)}+\sum_{1 \leq b<a \leq \nu} \sum_{C \in \mathbb{C}_{a}} \sum_{D \in \mathbb{C}_{b}}\left(\prod_{i \in C \cup D} q_{i}-\prod_{i \in C} q_{i} \prod_{i \in D} q_{i}\right)
\end{aligned}
$$

respectively.

Proof. The upper bound $U_{C B}^{(G)}$ follows immediately by a direct application of (6) for the associated r.v.'s $\delta_{C}\left(\mathbb{C}_{s}\right), s=1,2, \ldots, \nu$ (c.f. proof of Theorem 2); note also that

$$
\sum_{1 \leq b<a \leq \nu} \operatorname{Cov}\left(\delta_{C}\left(\mathbb{C}_{a}\right), \delta_{C}\left(\mathbb{C}_{b}\right)\right)=\sum_{1 \leq b<a \leq \nu}\left(R_{C}\left(\mathbb{C}_{a} \cup \mathbb{C}_{b}\right)-R_{C}\left(\mathbb{C}_{a}\right) R_{C}\left(\mathbb{C}_{b}\right)\right)
$$

Likewise, in order to derive the lower bound $L_{C B}^{(G)}$ it suffices to recall that $R=1-E\left(\prod_{s=1}^{\mu}(1-\right.$ $\left.\left.\delta_{P}\left(\mathbb{P}_{s}\right)\right)\right)$ and apply $(6)$ for the associated r.v.'s $1-\delta_{P}\left(\mathbb{P}_{s}\right), s=1,2, \ldots, \mu$.

Observe next that the non-negative integer valued r.v.'s $Y_{s}=\sum_{C \in \mathbb{C}_{s}} \prod_{i \in C}\left(1-X_{i}\right), s=$ $1,2, \ldots, \nu$, are associated (by virtue of $\mathrm{P} 4$ and $\mathrm{P} 5$ ) and vanish if and only if $\delta_{C}\left(\mathbb{C}_{s}\right)=1$. Hence

$$
\operatorname{Cov}\left(\delta_{C}\left(\mathbb{C}_{a}\right), \delta_{C}\left(\mathbb{C}_{b}\right)\right)=P\left(Y_{a}=0, Y_{b}=0\right)-P\left(Y_{a}=0\right) P\left(Y_{b}=0\right)
$$

and applying Theorem 1 we deduce

$$
\operatorname{Cov}\left(\delta_{C}\left(\mathbb{C}_{a}\right), \delta_{C}\left(\mathbb{C}_{b}\right)\right) \leq \operatorname{Cov}\left(Y_{a}, Y_{b}\right)=\sum_{C \in \mathbb{C}_{a}} \sum_{D \in \mathbb{C}_{b}}\left(\prod_{i \in C \cup D} q_{i}-\prod_{i \in C} q_{i} \prod_{i \in D} q_{i}\right), b<a
$$


which offers an upper bound for the double sum appearing in the $U_{C B}^{(G)}$. The lower bound for $L_{C B}^{(G)}$ is easily established by employing an analogous reasoning.

A careful inspection of the above proof reveals that bounds (9),(11) remain valid under the weaker assumption of association between $X_{i}, i \in I$.

It is also worth stressing that, usually the multiple summations involved in Theorem 3 contain a lot of vanishing summands. To be more specific, introducing the index sets

$$
\begin{aligned}
& B_{a}=\left\{j:\left(\cup_{C \in \mathbb{C}_{j}} C\right) \cap\left(\cup_{C \in \mathbb{C}_{a}} C\right) \neq \emptyset, j<a\right\}, a=2,3, \ldots, \nu \\
& B_{a}^{\prime}=\left\{j:\left(\cup_{P \in \mathbb{P}_{j}} P\right) \cap\left(\cup_{P \in \mathbb{P}_{a}} P\right) \neq \emptyset, j<a\right\}, a=2,3, \ldots, \mu
\end{aligned}
$$

(convention: $B_{1}=B_{1}^{\prime}=\emptyset$ ) one can easily verify that for $b \notin B_{a}, b<a$,

$$
R_{C}\left(\mathbb{C}_{a} \cup \mathbb{C}_{b}\right)=R_{C}\left(\mathbb{C}_{a}\right) R_{C}\left(\mathbb{C}_{b}\right), \quad 1-R_{P}\left(\mathbb{P}_{a} \cup \mathbb{P}_{b}\right)=\left(1-R_{P}\left(\mathbb{P}_{a}\right)\right)\left(1-R_{P}\left(\mathbb{P}_{b}\right)\right)
$$

Therefore the double summations of Theorem 3 could be restricted over $2 \leq a \leq \mu, b \in B_{a}^{\prime}$ for the lower bound and $2 \leq a \leq \nu, b \in B_{a}$ for the upper bound.

The next theorem consists a generalization of the bounds established by $\mathrm{Fu}$ and Koutras (1995). The development of the new bounds was made feasible by exploiting a proper modification of an idea used by Glaz and Naus (1991) to generate upper bounds for scan statistic probabilities. In order to state our result we need the following notation: For given partitions $\mathcal{C}$ and $\mathcal{P}$ we denote by $\mathbb{C}_{s}^{*}, \mathbb{P}_{s}^{*}$ the next two collections of minimal cut and path sets

$$
\mathbb{C}_{s}^{*}=\left\{D \in \cup_{l<s} \mathbb{C}_{l}: D \cap\left(\cup_{C \in \mathbb{C}_{s}} C\right) \neq \emptyset\right\}, s=1,2, \ldots, \nu
$$

and

$$
\mathbb{P}_{s}^{*}=\left\{D \in \cup_{l<s} \mathbb{P}_{l}: D \cap\left(\cup_{P \in \mathbb{P}_{s}} P\right) \neq \emptyset\right\}, s=1,2, \ldots, \mu
$$

$\left(\mathbb{C}_{1}^{*}=\mathbb{P}_{1}^{*}=\emptyset\right)$. As an illustration of the above definition consider a consecutive 3-out-of10 system (for a review on consecutive $k$-out-of- $n$ systems refer to Chao et al. (1995) or Papastavridis and Koutras (1993)). In this case $I=\{1,2, \ldots, 10\}, \mathbb{C}=\{\{i, i+1, i+2\}: i=$ $1,2, \ldots, 8\}$ and considering the following partition of $\mathbb{C}$ :

$$
\begin{aligned}
& \mathbb{C}_{1}=\{\{1,2,3\},\{2,3,4\},\{3,4,5\}\}, \mathbb{C}_{2}=\{\{4,5,6\},\{5,6,7\},\{6,7,8\}\}, \\
& \mathbb{C}_{3}=\{\{7,8,9\},\{8,9,10\}\}
\end{aligned}
$$

we deduce $\mathbb{C}_{1}^{*}=\emptyset$ and

$$
\begin{aligned}
\mathbb{C}_{2}^{*} & =\left\{D \in \mathbb{C}_{1}: D \cap\left(\cup_{C \in \mathbb{C}_{2}} C\right) \neq \emptyset\right\}=\left\{D \in \mathbb{C}_{1}: D \cap\{4,5,6,7,8\} \neq \emptyset\right\} \\
& =\{\{2,3,4\},\{3,4,5\}\}, \\
\mathbb{C}_{3}^{*} & =\left\{D \in \mathbb{C}_{1} \cup \mathbb{C}_{2}: D \cap\left(\cup_{C \in \mathbb{C}_{3}} C\right) \neq \emptyset\right\}=\left\{D \in \mathbb{C}_{1} \cup \mathbb{C}_{2}: D \cap\{7,8,9,10\} \neq \emptyset\right\} \\
& =\{\{5,6,7\},\{6,7,8\}\} .
\end{aligned}
$$


Theorem 4 The reliability $R$ of any coherent structure is bounded below and above by

$$
\begin{aligned}
L_{F K}^{(G)} & =1-\left(1-R_{P}\left(\mathbb{P}_{1}\right)\right) \prod_{s=2}^{\mu}\left(1-\left(R_{P}\left(\mathbb{P}_{s}^{*} \cup \mathbb{P}_{s}\right)-R_{P}\left(\mathbb{P}_{s}^{*}\right)\right)\right) \\
& =1-\left(1-R_{P}\left(\mathbb{P}_{1}\right)\right) \prod_{s=2}^{\mu}\left(1-E\left(\delta_{P}\left(\mathbb{P}_{s}\right)\left(1-\delta_{P}\left(\mathbb{P}_{s}^{*}\right)\right)\right)\right)
\end{aligned}
$$

and

$$
\begin{aligned}
U_{F K}^{(G)} & =R_{C}\left(\mathbb{C}_{1}\right) \prod_{s=2}^{\nu}\left(1-\left(R_{C}\left(\mathbb{C}_{s}^{*}\right)-R_{C}\left(\mathbb{C}_{s}^{*} \cup \mathbb{C}_{s}\right)\right)\right) \\
& =R_{C}\left(\mathbb{C}_{1}\right) \prod_{s=2}^{\nu}\left(1-E\left(\left(1-\delta_{C}\left(\mathbb{C}_{s}\right)\right) \delta_{C}\left(\mathbb{C}_{s}^{*}\right)\right)\right)
\end{aligned}
$$

respectively.

Proof. To establish the upper bound $U_{F K}^{(G)}$, observe first that for $s=1,2, \ldots, \nu$ we have

$$
R_{C}\left(\cup_{j \leq s-1} \mathbb{C}_{j}\right)-R_{C}\left(\cup_{j \leq s} \mathbb{C}_{j}\right)=E\left(\left(1-\delta_{C}\left(\mathbb{C}_{s}\right)\right) \prod_{j=1}^{s-1} \delta_{C}\left(\mathbb{C}_{j}\right)\right)
$$

or alternatively,

$$
R_{C}\left(\cup_{j \leq s} \mathbb{C}_{j}\right)=R_{C}\left(\cup_{j \leq s-1} \mathbb{C}_{j}\right)-E\left(\left[\left(1-\delta_{C}\left(\mathbb{C}_{s}\right)\right) \delta_{C}\left(\mathbb{C}_{j}^{*}\right)\right]\left[\delta_{C}\left(\cup_{j \leq s-1} \mathbb{C}_{j} \backslash \mathbb{C}_{s}^{*}\right)\right]\right)
$$

Conditioning on $\mathcal{F}_{s}=\sigma\left(X_{i}, i \in \cup_{C \in \mathbb{C}_{s}} C\right)$, the bracketed terms inside the mean value operator become coordinatewise non-decreasing functions of the independent r.v.'s $X_{i}, i \in I \backslash \cup_{C \in \mathbb{C}_{s}} C$. Hence they are associated (by P2, P3, P4) and with the aid of (6) we may write

$$
E\left(\left(1-\delta_{C}\left(\mathbb{C}_{s}\right)\right) \prod_{j=1}^{s-1} \delta_{C}\left(\mathbb{C}_{j}\right) \mid \mathcal{F}_{s}\right) \geq E\left(\left(1-\delta_{C}\left(\mathbb{C}_{s}\right)\right) \delta_{C}\left(\mathbb{C}_{s}^{*}\right) \mid \mathcal{F}_{s}\right) E\left(\delta_{C}\left(\cup_{j \leq s-1} \mathbb{C}_{j} \backslash \mathbb{C}_{s}^{*}\right) \mid \mathcal{F}_{s}\right)
$$

Since $\delta_{C}\left(\cup_{j \leq s-1} \mathbb{C}_{j} \backslash \mathbb{C}_{s}^{*}\right)$ is independent of $X_{i}, i \in \bigcup_{C \in \mathbb{C}_{s}} C$, we conclude that

$$
E\left(\delta_{C}\left(\cup_{j \leq s-1} \mathbb{C}_{j} \backslash \mathbb{C}_{s}^{*}\right) \mid \mathcal{F}_{s}\right)=E\left(\delta_{C}\left(\cup_{j \leq s-1} \mathbb{C}_{j} \backslash \mathbb{C}_{s}^{*}\right)\right) \geq E\left(\delta_{C}\left(\cup_{j \leq s-1} \mathbb{C}_{j}\right)\right)=R_{C}\left(\cup_{j \leq s-1} \mathbb{C}_{j}\right) .
$$

Using the above inequality in (15) and applying next the mean value operator to the resulting formula we get

$$
E\left(\left(1-\delta_{C}\left(\mathbb{C}_{s}\right)\right) \prod_{j=1}^{s-1} \delta_{C}\left(\mathbb{C}_{j}\right)\right) \geq E\left(\left(1-\delta_{C}\left(\mathbb{C}_{s}\right)\right) \delta_{C}\left(\mathbb{C}_{s}^{*}\right)\right) R_{C}\left(\cup_{j \leq s-1} \mathbb{C}_{j}\right)
$$


This may be used in conjunction with (14) to verify that

$$
\begin{aligned}
R_{C}\left(\cup_{j \leq s} \mathbb{C}_{j}\right) & \leq R_{C}\left(\cup_{j \leq s-1} \mathbb{C}_{j}\right)\left(1-E\left(\left(1-\delta_{C}\left(\mathbb{C}_{s}\right)\right) \delta_{C}\left(\mathbb{C}_{s}^{*}\right)\right)\right) \\
& =R_{C}\left(\cup_{j \leq s-1} \mathbb{C}_{j}\right)\left(1-\left(R_{C}\left(\mathbb{C}_{s}^{*}\right)-R_{C}\left(\mathbb{C}_{s} \cup \mathbb{C}_{s}^{*}\right)\right)\right)
\end{aligned}
$$

The upper bound $U_{F K}^{(G)}$ is now easily obtained by induction on $s$.

For the establishment of the lower bound $L_{F K}^{(G)}$ one can proceed in a similar manner as above or appeal to duality arguments for cut and path sets.

In some situations, the evaluation of the bounds provided by Theorem 4 turn out to be quite involved. Nevertheless, it is the rule rather that the exception that, after the determination of the families $\mathbb{C}_{s}^{*}, \mathbb{P}_{s}^{*}$, one can easily spot out binary functions $a_{s}=a_{s}\left(X_{i}, i \in\right.$ $I), s=1,2, \ldots, \nu$ and $a_{s}^{\prime}=a_{s}^{\prime}\left(X_{i}, i \in I\right), s=1,2, \ldots, \mu$ with the following properties:

(i) $a_{s}$ is independent of $\delta_{C}\left(\mathbb{C}_{s}\right)$ and $a_{s}^{\prime}$ is independent of $\delta_{P}\left(\mathbb{P}_{s}\right)$.

(ii) $a_{s} \leq \delta_{C}\left(\mathbb{C}_{s}^{*}\right)$ and $a_{s}^{\prime} \geq \delta_{P}\left(\mathbb{P}_{s}^{*}\right)$.

Should this be the case, the following corollary could be employed to gain simpler (but occasionally weaker) bounds than the ones suggested by Theorem 4 .

Corollary 1 If there exist binary functions $a_{s}, a_{s}^{\prime}$ satisfying conditions (i) and (ii) then the reliability $R$ of the system is bounded below and above as follows

$1-\left(1-R_{P}\left(\mathbb{P}_{1}\right)\right) \prod_{s=2}^{\mu}\left(1-P\left(a_{s}^{\prime}=0\right) R_{P}\left(\mathbb{P}_{s}\right)\right) \leq R \leq R_{C}\left(\mathbb{C}_{1}\right) \prod_{s=2}^{\nu}\left(1-P\left(a_{s}=1\right)\left(1-R_{C}\left(\mathbb{C}_{s}\right)\right)\right)$.

Proof. Conditions (i) and (ii) guarantee that

$$
\begin{aligned}
E\left(\left(1-\delta_{C}\left(\mathbb{C}_{s}\right)\right) \delta_{C}\left(\mathbb{C}_{s}^{*}\right)\right) & \geq E\left(\left(1-\delta_{C}\left(\mathbb{C}_{s}\right)\right) a_{s}\right)=E\left(1-\delta_{C}\left(\mathbb{C}_{s}\right)\right) E\left(a_{s}\right) \\
& =\left(1-R_{C}\left(\mathbb{C}_{s}\right)\right) P\left(a_{s}=1\right) .
\end{aligned}
$$

The proof of the RHS inequality is now easily completed by exploiting Theorem's 4 upper bound $U_{F K}^{(G)}$. A similar reasoning (or alternatively, duality arguments) may be used to establish the LHS inequality.

Needless to say, if the evaluation of the quantities $P\left(a_{s}=1\right)=E\left(a_{s}\right)$ or $P\left(a_{s}^{\prime}=0\right)=$ $1-E\left(a_{s}^{\prime}\right)$ is intractable, a good practice would be to replace $E\left(a_{s}\right)$ by an appropriate lower bound and $E\left(a_{s}^{\prime}\right)$ by an appropriate upper bound (e.g. Esary and Proschan or covariance bounds).

It is worth mentioning that in the extreme case $\nu=N, \mathbb{C}_{s}=\left\{C_{s}\right\}, s=1,2, \ldots, \nu$ and $\mu=M, \mathbb{P}_{s}=\left\{P_{s}\right\}, s=1,2, \ldots, \mu$, the binary functions $a_{s}, a_{s}^{\prime}$ coincide with the $a$-cut and $a$-path structures introduced by Koutras et al. (1996) and the bounds of Corollary 1 reduce to the ones derived in the same article.

Another pertinent remark is that conditions (i) and (ii) do not lead to a uniquely determined family of binary functions $a_{s}$ and $a_{s}^{\prime}$. Nevertheless, it is not difficult to verify that the optimum choice for them (in terms of upper and lower bounds' quality) is offered by 
- $a_{s}=1$ if $\mathbb{C}_{s}^{*}=\emptyset$ and $a_{s}=\delta_{C}\left(\operatorname{minimal}\left\{C \backslash\left(\cup_{D \in \mathbb{C}_{s}} D\right): C \in \mathbb{C}_{s}^{*}\right\}\right)$ otherwise, $s=1, \ldots, \nu$,

- $a_{s}^{\prime}=0$ if $\mathbb{P}_{s}^{*}=\emptyset$ and $a_{s}^{\prime}=\delta_{P}\left(\operatorname{minimal}\left\{P \backslash\left(\cup_{Q \in \mathbb{P}_{s}} Q\right): P \in \mathbb{P}_{s}^{*}\right\}\right)$ otherwise, $s=1, \ldots, \mu$,

where minimal $(\mathbb{A})$ denotes the set of all minimal sets of $\mathbb{A}$ with respect to the partial ordering $\subset$. (Note that, if $\emptyset \in \mathbb{A}$, then $\operatorname{minimal}(\mathbb{A})=\{\emptyset\} ;$ in that case we have $\delta_{C}(\operatorname{minimal}(\mathbb{A}))$ $=\delta_{C}(\{\emptyset\})=1-\prod_{i \in \emptyset}\left(1-X_{i}\right)=0$ and $\left.\delta_{P}(\operatorname{minimal}(\mathbb{A}))=\delta_{P}(\{\emptyset\})=1\right)$.

Closing the present paragraph we mention the following partition-based generalization of the min-max bounds (5)

$$
\max _{1 \leq s \leq \mu} R_{P}\left(\mathbb{P}_{s}\right) \leq R \leq \min _{1 \leq s \leq \nu} R_{C}\left(\mathbb{C}_{s}\right)
$$

(the proof is left to the reader). Since these bounds exhibit very poor performance we are not going to consider them in the sequel.

\section{Application}

In this section we demonstrate how the generalized reliability bounds can be fruitfully applied to a specific structure which has recently attracted considerable research interest: the twodimensional consecutive- $k_{1} \times k_{2}$-out-of- $n_{1} \times n_{2}$ system. It is worth stressing that in such a system the reliability evaluation by exact formulae is highly involved.

The two-dimensional consecutive- $k_{1} \times k_{2}$-out-of- $n_{1} \times n_{2}$ system consists of $n_{1} n_{2}$ components arranged in a square grid of size $n_{1} \times n_{2}$ and fails if and only if there exists at least one square of size $k_{1} \times k_{2}\left(1<k_{1} \leq n_{1}, 1<k_{2} \leq n_{2}\right)$ with all its $k_{1} k_{2}$ components failed. It was first introduced by Salvia and Lasher (1990) who also discussed several practical applications of the system to real life problems.

As Yamamoto and Miyakawa (1995) pointed out, the evaluation of the exact reliability of a general two-dimensional consecutive- $k_{1} \times k_{2}$-out-of- $n_{1} \times n_{2}$ system is a very formidable task; for example, the computing time for assessing the reliability of it is of order $O\left(k_{2}^{n_{1}-k_{1}} n_{1}^{2} k_{1} n_{2}\right)$ while the memory requirements for implementing their algorithm are as high as $\left(n_{1}-k_{1}+1\right) 2^{n_{1}-k_{1}+2}$ bits and $2 k_{2}^{n_{1}-k_{1}+1}$ real numbers. In the last few years, several bounds and approximations for system's reliability have been proposed by Salvia and Lasher (1990), Koutras and Papastavridis (1993), Koutras et al. (1993, 1996), Fu and Koutras (1994, 1995), Yamamoto and Miyakawa (1995), Barbour et al. (1996), Makri and Psillakis (1997), and Godbole et al. (1998).

Let us label the $n_{1} n_{2}$ components of the system as $(i, j), i=1,2, \ldots, n_{1}, j=1,2, \ldots, n_{2}$ and denote by $X_{(i, j)}$ and $q_{(i, j)}=1-p_{(i, j)}$ or simply by $X_{i j}$ and $q_{i j}=1-p_{i j}$ the state and failure probability of component $(i, j)$ respectively. If we introduce the notation

$$
I=\left\{(i, j): i=1,2, \ldots, n_{1}, j=1,2, \ldots, n_{2}\right\}, N_{1}=n_{1}-k_{1}+1, N_{2}=n_{2}-k_{2}+1, N=N_{1} N_{2},
$$

it is clear that the minimal cut sets of the system will be given by

$C_{(i, j)}=\left\{(u, v) \in I, u=i, i+1, \ldots, i+k_{1}-1, v=j, j+1, \ldots, j+k_{2}-1\right\}, i=1, \ldots, N_{1}, j=1, \ldots, N_{2}$. 
(Allowing an abuse of the notation we shall also write $C_{i j}$ instead of $C_{(i, j)}$ ). Throughout we shall use the notation $\left(a_{1}, b_{1}\right)<\left(a_{2}, b_{2}\right)$ to indicate that $a_{1}<a_{2}$ or $a_{1}=a_{2}, b_{1}<b_{2}$ (lexicographic ordering).

i) Generalized Esary and Proschan Bounds. The lower EP bound (2) reads

$$
L_{E P}=\prod_{C \in \mathbb{C}}\left(1-\prod_{a \in C} q_{a}\right)=\prod_{i=1}^{N_{1}} \prod_{j=1}^{N_{2}}\left(1-\prod_{u=i}^{i+k_{1}-1} \prod_{v=j}^{j+k_{2}-1} q_{u v}\right)
$$

and in the iid case $p_{i j}=p, q_{i j}=q$ it reduces to $L_{E P}=\left(1-q^{k_{1} k_{2}}\right)^{N}$.

Manifestly, the development of generalized EP bounds depends on the specific selection of the partition $\mathcal{C}$ of $\mathbb{C}$. Taking into account the remarks following Theorem 2 , we should try to balance between the quality of the bounds (thicker partitions) and the computational convenience of them (thinner partitions). It seems that, for the system under consideration, a reasonable choice for $\mathcal{C}$ is offered by $\mathcal{C}=\left\{\mathbb{C}_{s}, s=1,2, \ldots, N_{1}\right\}$ where $\mathbb{C}_{s}=\left\{C_{s j}, j=\right.$ $\left.1,2, \ldots, N_{2}\right\}$. In this case we have

$$
\begin{aligned}
R_{C}\left(\mathbb{C}_{s}\right) & =E\left(\prod_{C \in \mathbb{C}_{s}}\left(1-\prod_{a \in C}\left(1-X_{a}\right)\right)\right)=E\left(\prod_{j=1}^{N_{2}}\left(1-\prod_{u=s}^{s+k_{1}-1} \prod_{i=j}^{j+k_{2}-1}\left(1-X_{u i}\right)\right)\right) \\
& =E\left(\prod_{j=1}^{N_{2}}\left(1-\prod_{i=j}^{j+k_{2}-1}\left(1-Y_{i}(s)\right)\right)\right)
\end{aligned}
$$

where $\left.Y_{i}(s)=1-\prod_{u=s}^{s+k_{1}-1}\left(1-X_{u i}\right)\right)$. Since the r.v.'s $Y_{i}(s), i=1,2, \ldots, n_{2}$ are independent, it is evident that $R_{C}\left(\mathbb{C}_{s}\right)$ coincides with the reliability of a consecutive $k_{2}$-out-of- $n_{2}$ system with component reliabilities

$$
E\left(Y_{i}(s)\right)=1-E\left(\prod_{u=s}^{s+k_{1}-1}\left(1-X_{u i}\right)\right)=1-\prod_{u=s}^{s+k_{1}-1} q_{u i}, i=1,2, \ldots, n_{2} .
$$

(For a review on consecutive $k$-out-of- $n$ systems and methods for the evaluation of their reliability function via simple recursive relations we refer to Chao et al. (1995) or Papastavridis and Koutras (1993)). Therefore, if we denote by $R_{C(n, k)}\left(p_{1}, \ldots, p_{n}\right)$ the reliability function of a consecutive $k$-out-of- $n$ system with component reliabilities $p_{1}, p_{2}, \ldots, p_{n}$ $\left(R_{C(n, k)}(p, \ldots, p) \equiv R_{C(n, k)}(p)\right)$, then the generalized Esary and Proschan lower bound will take on the form

$$
L_{E P}^{(G)}=\prod_{s=1}^{n_{1}-k_{1}+1} R_{C}\left(\mathbb{C}_{s}\right)=\prod_{s=1}^{n_{1}-k_{1}+1} R_{C\left(n_{2}, k_{2}\right)}\left(1-\prod_{u=s}^{s+k_{1}-1} q_{u 1}, \ldots, 1-\prod_{u=s}^{s+k_{1}-1} q_{u n_{2}}\right)
$$

In the iid case we have $E\left(Y_{i}(s)\right)=1-q^{k_{1}}$ and the aforementioned lower bound simplifies to $L_{E P}^{(G)}=\left(R_{C\left(n_{2}, k_{2}\right)}\left(1-q^{k_{1}}\right)\right)^{N_{1}}$. This last bound has been for the first time mentioned by Yamamoto and Miyakawa (1995) . 
ii) Covariance Bounds. Applying formula (4) we deduce the covariance bound

$U_{C B}=L_{E P}+\sum_{j<s}\left(\prod_{i \in C_{s} \cup C_{j}} q_{i}-\prod_{i \in C_{s}} q_{i} \prod_{i \in C_{j}} q_{i}\right)=L_{E P}+\sum_{s \in I_{0}} \sum_{j \in B_{s}}\left(\prod_{i \in C_{s} \cup C_{j}} q_{i}-\prod_{i \in C_{s}} q_{i} \prod_{i \in C_{j}} q_{i}\right)$

where $I_{0}=\left\{(i, j): i=1,2, \ldots, N_{1}, j=1,2, \ldots, N_{2}\right\}, B_{s}=\left\{j: j<s, C_{s} \cap C_{j} \neq \emptyset\right\}$. In the iid case it can be easily verified that the double sum reads

$$
\begin{aligned}
& \sum_{s \in I_{0}} \sum_{j<s: C_{s} \cap C_{j} \neq \emptyset}\left(q^{\left|C_{s}\right|+\left|C_{j}\right|-\left|C_{s} \cap C_{j}\right|}-q^{\left|C_{s}\right|+\left|C_{j}\right|}\right) \\
\leq & \left(N_{1}-1\right)\left(N_{2}-1\right) \sum_{i=1}^{k_{1}-1} \sum_{j=1}^{k_{2}-1} 2\left(q^{2 k_{1} k_{2}-i j}-q^{2 k_{1} k_{2}}\right)+\left(N-N_{2}\right) \sum_{i=1}^{k_{1}-1}\left(q^{2 k_{1} k_{2}-i k_{2}}-q^{2 k_{1} k_{2}}\right) \\
& +\left(N-N_{1}\right) \sum_{j=1}^{k_{2}-1}\left(q^{2 k_{1} k_{2}-k_{1} j}-q^{2 k_{1} k_{2}}\right)
\end{aligned}
$$

A generalized covariance bound can be derived by considering the partition $\mathcal{C}$ introduced in 4.1(i). In this case, Theorem 3 implies the upper bound

$$
U_{C B}^{(G)} \leq L_{E P}^{(G)}+\sum_{s=2}^{N_{1}} \sum_{j=\max \left\{1, s-k_{1}+1\right\}}^{s-1} \sum_{a=1}^{N_{2}} \sum_{\substack{b=1 \\|b-a|<k_{2}}}^{N_{2}}\left(\prod_{i \in C_{s a} \cup C_{j b}} q_{i}-\prod_{i \in C_{s a}} q_{i} \prod_{i \in C_{j b}} q_{i}\right) .
$$

In the iid case, $U_{C B}^{(G)}$ can be bounded as follows

$$
\begin{aligned}
U_{C B}^{(G)} & \leq L_{E P}^{(G)}+\left(N_{1}-1\right) N_{2} \sum_{j=1}^{k_{1}-1} \sum_{b=1}^{2 k_{2}-1}\left(q^{\left|C_{k_{1} k_{2}} \cup C_{j b}\right|}-q^{2 k_{1} k_{2}}\right) \\
& =L_{E P}^{(G)}+\left(N_{1}-1\right) N_{2}\left(2 \sum_{j=1}^{k_{1}-1} \sum_{b=1}^{k_{2}-1} q^{2 k_{1} k_{2}-j b}+\sum_{j=1}^{k_{1}-1} q^{2 k_{1} k_{2}-j k_{2}}-\left(k_{1}-1\right)\left(2 k_{2}-1\right) q^{2 k_{1} k_{2}}\right)
\end{aligned}
$$

iii) Generalized FK Bounds. Considering the partition $\{\{C\}: C \in \mathbb{C}\}$, Theorem 4 gives birth to the upper bound

$$
U_{F K}^{(G)}=\left(1-\prod_{a \in C_{(1,1)}} q_{a}\right) \prod_{s \in I_{0} \backslash\{(1,1)\}}\left(1-E\left(\prod_{a \in C_{s}}\left(1-X_{a}\right) \prod_{b \in B_{s}}\left(1-\prod_{a \in C_{b}}\left(1-X_{a}\right)\right)\right)\right.
$$

where $B_{s}=\left\{b: C_{b} \cap C_{s} \neq \emptyset, b<s\right\}=\left\{b: C_{b} \in \mathbb{C}_{s}^{*}\right\}$. In general, the evaluation of the above bound is computationally demanding. However, it is much easier than the evaluation of the exact reliability itself since each of the $\left|I_{0} \backslash\{(1,1)\}\right|=N-1$ terms of the product involve at 
most $\left(3 k_{2}-2\right)\left(2 k_{1}-1\right)-\left(k_{2}-1\right)$ components instead of $n_{1} n_{2}$. In the case of dealing with iid two-dimensional consecutive- $2 \times 2$-out-of- $n_{1} \times n_{2}$ system, the above bound reads

$$
\begin{aligned}
U_{F K}^{(G)}= & \left(1-q^{4}\right)\left(1-q^{4}+q^{6}\right)^{n_{2}-k_{2}}\left(1-q^{4}+q^{6}+q^{7}-q^{8}\right)^{n_{1}-k_{1}} \\
& \times\left(1-q^{4}+2 q^{6}+2 q^{7}-4 q^{8}+q^{10}\right)^{\left(n_{1}-k_{1}\right)\left(n_{2}-k_{2}\right)}
\end{aligned}
$$

A simpler but less effective upper bound can be established by exploiting Corollary 1. More specifically, considering the optimum choice for the binary functions $a_{s}$ over the partition $\{\{C\}: C \in \mathbb{C}\}$, we deduce

$$
\begin{aligned}
& R_{C}\left(\mathbb{C}_{1}\right) \prod_{s=2}^{\nu}\left(1-R\left(\mathbb{C}_{s}^{* *}\right)\left(1-R_{C}\left(\mathbb{C}_{s}\right)\right)\right) \\
= & \left(1-\prod_{a \in C_{(1,1)}} q_{a}\right) \prod_{s \in I_{0} \backslash\{(1,1)\}}\left(1-E\left(\prod_{C \in \mathbb{C}_{s}^{* *}}\left(1-\prod_{a \in C}\left(1-X_{a}\right)\right)\right) \prod_{a \in C_{s}} q_{a}\right)
\end{aligned}
$$

where $\mathbb{C}_{s}^{* *}=\operatorname{minimal}\left\{C_{a} \backslash C_{s}: C_{a} \cap C_{s} \neq \emptyset, a<s\right\}$. Employing next the $E P$ lower bound (2) for the mean operator appearing in the RHS we gain the following upper bound

$$
U_{F K}^{(G *)}=\left(1-\prod_{a \in C_{(1,1)}} q_{a}\right) \prod_{s \in I_{0} \backslash\{(1,1)\}}\left(1-\prod_{C \in \mathbb{C}_{s}^{* *}}\left(1-\prod_{a \in C} q_{a}\right) \prod_{a \in C_{s}} q_{a}\right)
$$

In the iid case, $U_{F K}^{(G *)}$ can be easily checked to be upper bounded by

$$
\begin{aligned}
U_{F K}^{(G *)} \leq & \left(1-q^{k_{1} k_{2}}\right)\left(1-\left(1-q^{k_{1}}\right) q^{k_{1} k_{2}}\right)^{n_{2}-k_{2}}\left(1-\left(1-q^{k_{2}}\right) b q^{k_{1} k_{2}}\right)^{n_{1}-k_{1}} \\
& \times\left(1-\left(1-q^{k_{1}}\right)\left(1-q^{k_{2}}\right) b q^{k_{1} k_{2}}\right)^{n_{1}-k_{1}}\left(1-\left(1-q^{k_{1}}\right)\left(1-q^{k_{2}}\right) b^{2} q^{k_{1} k_{2}}\right)^{\left(n_{1}-k_{1}\right)\left(n_{2}-k_{2}-1\right)}
\end{aligned}
$$

where $b=\prod_{i=1}^{k_{1}-1} \prod_{j=1}^{k_{2}-1}\left(1-q^{k_{1} k_{2}-i j}\right)$.

It is worth noting that, on choosing the binary functions $a_{s}(\mathbf{X})=\prod_{a \in L_{s}} X_{a}, s \in I_{0}$ where

$$
\begin{aligned}
L_{(1,1)} & =\emptyset, L_{(1, j)}=\{(1, j-1)\}, j=2,3, \ldots, N_{2}, L_{(i, 1)}=\left\{\left(i-1, k_{2}\right)\right\}, i=2,3, \ldots, N_{1}, \\
L_{(i, j)} & =\left\{\left(i-1, j+k_{2}-1\right),(i, j-1)\right\}, j=2,3, \ldots, N_{2}, i=2,3, \ldots, N_{1},
\end{aligned}
$$

Corollary 1 will result in the following (ordinary) FK bound

$$
\begin{aligned}
U_{F K}= & \left(1-\prod_{a \in C_{11}} q_{a}\right) \prod_{j=2}^{N_{2}}\left(1-p_{1, j-1} \prod_{a \in C_{1 j}} q_{a}\right) \prod_{i=2}^{N_{1}}\left(1-p_{i-1, k_{2}} \prod_{a \in C_{i 1}} q_{a}\right) \\
& \times \prod_{i=2}^{N_{1}} \prod_{j=2}^{N_{2}}\left(1-p_{i-1, j+k_{2}-1} p_{i, j-1} \prod_{a \in C_{i j}} q_{a}\right)
\end{aligned}
$$

which, in the iid case, simplifies to $U_{F K}=\left(1-q^{k_{1} k_{2}}\right)\left(1-p q^{k_{1} k_{2}}\right)^{N_{1}+N_{2}-2}\left(1-p^{2} q^{k_{1} k_{2}}\right)^{\left(N_{1}-1\right)\left(N_{2}-1\right)}$. 
It is noteworthy that, applying Corollary 1 to the partition $\mathcal{C}$ (see (i)) we may obtain a generalized $F K$ bound of the form

$$
U_{Y M}=R_{C\left(n_{2}, k_{2}\right)}\left(1-q^{k_{1}}\right)\left(1-R_{C\left(n_{2}, k_{2}\right)}(1-q)\left(1-R_{C\left(n_{2}, k_{2}\right)}\left(1-q^{k_{1}}\right)\right)\right)^{N_{1}-1} .
$$

Such a bound has also been given (without stating the details of the proof) by Yamamoto and Miyakawa (1995).

In Tables 1-5 we present, for a variety of choices of $n_{1}, n_{2}, k_{1}, k_{2}, p_{i j}$, the values of the bounds described in formulae (16), (17), (18), (20), (21), (23), (24) (they have been labeled as $L_{E P}, L_{E P}^{G}, U_{C B}, U_{C B}^{G}, U_{F K}^{G *}, U_{F K}, U_{Y M}$ respectively. In the iid cases we use (19), (22) instead of (18), (21) respectively). In Tables $1,2,5$ which refer to the case $n_{1}=n_{2}, k_{1}=k_{2}$, a number of previously established bounds has been included for comparison reasons (although these bounds could possibly be extended to cover the case $n_{1} \neq n_{2}, k_{1} \neq k_{2}$, no explicit formulae have appeared in the related bibliography so far): LBonf and UBonf refer to the Bonferroni-type bounds suggested by Makri and Psillakis(1997), LPo and UPo to the Chen-Stein bounds developed by Koutras et al.(1993) (actually, we use the slightly improved formula (6) in Barbour et al. (1996)), UJ to the upper Janson's-inequality-based bounds which were studied by Godbole et al. (1998) (c.f. middle term of formula (6) therein) and LCP, UCP to the compound Poisson approximation based bounds of Barbour et al. (1996) (the last bounds were computed by formula (8) in Barbour et al.(1996) and not (10) as was done by Godbole et al.(1998) since the latter exhibits very poor performance as compared to the first one. Note also that in formula (8) the upper limits of the double sum should read $k-1$ instead of $k$ ). Column labeled as $R_{\text {sim }}$ contains the simulated value of the exact reliability based on $10^{5}$ iterations).

In Tables 1-3 several iid cases were examined whereas Tables 4, 5 deal with the following non-iid case: $q_{i j}=1-p_{i j}=1 /|i-j|$ if $|i-j|>1$ and $q_{i j}=0.5$ otherwise.

The boldface figures indicate the best available (lower and upper) bound for each specific choice of the parameters. Note that, some figures are negative or larger than one. Although, in this case, the correct values are 0 and 1 respectively, we have recorded the actual numerical quantity deduced by the mathematical formula at hand. An overall performance measure of each individual bound is provided by the row labeled m.e. which contains the mean absolute error (over the $p$ values indicated by the tables) with respect to the simulated reliability values. In the calculation of the errors, negative lower bounds were replaced by 0 while upper bounds exceeding 1 were replaced by 1 .

From the Tables it is clear that the generalized $E P$ lower bound $L_{E P}^{G}$ and generalized $F K$ upper bound $U_{F K}^{G *}$ exhibit the highest performance among the bounds available in the literature so far. As a matter of fact $U_{F K}^{G *}$ is uniformly better for the whole range of parameters considered here, whereas $L_{E P}^{G}$ is only occasionally beaten by $L C P$ chiefly for high component survival probabilities (observe however that, in these cases, there is a very slight discrepancy between $L C P$ and $L_{E P}^{G}$ ). It is also worth stressing that the exceptional performance of $L_{E P}^{G}, U_{F K}^{G *}$ is achieved without having to resort to computationally intractable schemes. In order to help the practically minded reader grasp the usefulness of the aforementioned bounds we recall that for $n_{1}=n_{2}=50, k_{1}=k_{2}=3$ the exact reliability evaluation by 
the Yamamoto and Miyakawa (1995) algorithm would require computing time for a single $p$ value of order $O\left(3^{48} \times 50^{3}\right)$, the memory requirements being $48 \times 2^{49}$ bits and $2 \times 3^{48}$ real numbers. Nevertheless, the calculation of all the figures shown in Table 1 (except from the simulated reliability values which are time consuming) was performed on a $350 \mathrm{MHz}$ Pentium II PC in less than one second. This time was achieved by using Mathematica for Windows and could be substantially reduced should we decide to use a faster high level language (e.g. $\mathrm{C})$.

\section{References}

[1] Barbour, A.D., Chryssaphinou, O. and Roos, M. (1996). Compound Poisson approximation in systems reliability. Naval Research Logistics 43, 251-264.

[2] Barlow, R.E. and Proschan, F. (1981). Statistical Theory of Reliability and Life Testing. To Begin with, Silver Spring, MD.

[3] Boutsikas, M.V. And Koutras, M.V. (1999). A bound for the distribution of the sum of discrete associated or NA random variables. Annals of Applied Probability (to appear).

[4] Chao, M.T., Fu J.C. And Koutras, M.V. (1995). Survey of Reliability studies of consecutive- $k$-out-of- $n: F$ and related systems. IEEE Transactions on Reliability 44, $120-127$.

[5] Esary, J.D. and Proschan, F. (1963) Coherent structures of non-identical components. Technometrics 5, 191-209.

[6] Esary, J.D., Proschan, F. and Walkup, D.W. (1967). Association of random variables, with applications. Annals of Mathematical Statistics 38, 1466-1474.

[7] Fu, J.C. And Koutras, M.V. (1994). Poisson approximations for two-dimensional patterns. Annals of the Institute of Statistical Mathematics 46, 179-192.

[8] Fu, J.C. And Koutras, M.V. (1995). Reliability bounds for coherent structures with independent components. Statistics \& Probability Letters 22, 137-148.

[9] Glaz, J. And Naus, J.I. (1991). Tight bounds and approximations for scan statistic probabilities for discrete data. The Annals of Applied Probability 1, 306-318.

[10] Godbole, A.P., Potter, L.K. And Sklar, J.K. (1998). Improved upper bounds for the reliability of $d$-dimensional consecutive- $k$-out-of- $n: F$ systems. Naval Research Logistics 45, 219-230.

[11] Koutras, M.V. and Papastavridis, S.G. (1993). Application of the Chen-Stein method for bounds and limit theorems in the reliability of coherent structures. Naval Research Logistics 40, 617-631. 
[12] Koutras, M.V., Papastavridis, S.G. and Papadopoulos, G.K. (1993). Reliability of a 2-dimensional consecutive- $k$-out-of- $n: F$ systems. IEEE Transactions on Reliability 42, 658-661.

[13] Koutras, M.V., Papastavridis, S.G. and Petakos, K.I. (1996). Bounds for coherent reliability structures. Statistics $\&$ Probability Letters 26, 431-448.

[14] Makri, F.S. ANd Psillakis, Z.M. (1997). Bounds for reliability of $k$-within 2dimensional consecutive- $r$-out-of- $n$ failure systems. Microelectronics and Reliability $\mathbf{3 7}$, $1217-1224$.

[15] Papastavridis, S.G. and Koutras, M.V. (1993). Consecutive- $k$-out-of- $n$ Systems. New Trends in System Reliability Evaluation (Ed. Misra, K.B.), 228-248. Elsevier Science Pub. B.V., Amsterdam.

[16] Salvia, A. And Lasher, W. (1990). 2-dimensional consecutive- $k$-out-of- $n$ : $F$ models. IEEE Transactions on Reliability 39, 382-385.

[17] Yamamoto, H. and Miyakawa, M. (1995). Reliability of a linear connected- $(r, s)-$ out-of- $(m, n): F$ lattice system. IEEE Transactions on Reliability 44, 333-336. 
Table 1, $n_{1}=50, n_{2}=50, k_{1}=3, k_{2}=3$ (iid)

\begin{tabular}{|c|c|c|c|c|c|c|c|c|c|c|c|c|c|c|c|}
\hline p & LBonf & LPo & $L C P$ & $L_{E P}$ & $L_{E P}{ }^{G}$ & $\boldsymbol{R}_{\text {sim }}$ & $U_{F K}{ }^{G^{*}}$ & $\overline{U_{F K}}$ & $\overline{U_{C B}}$ & $U_{C B}{ }^{G}$ & $U_{Y M}$ & $U J$ & UCP & UPo & UBonf \\
\hline .5 & -3.49 & $\begin{array}{l}-.794 \\
\end{array}$ & -1.77 & .0111 & .0188 & .0326 & .0431 & .3095 & 1.587 & 9999 & .8631 & .0621 & 1.831 & 8165 & 2710 \\
\hline .55 & -.742 & -.260 & -.109 & .1748 & .2036 & .2375 & .2537 & .5795 & .5913 & .4547 & .8975 & .2723 & .5740 & 6098 & .4507 \\
\hline .6 & .3963 & .3945 & .5319 & .5466 & .5675 & .5893 & .5966 & .7997 & 6417 & .6229 & .9282 & .6037 & 6420 & 6988 & 6733 \\
\hline .65 & 8184 & .7994 & .8390 & .8339 & .8403 & .8476 & .8483 & 9245 & 8520 & .8505 & .9593 & 8497 & 8535 & 8685 & 8530 \\
\hline .7 & 9547 & .9502 & .9572 & .9557 & .9568 & .9580 & .9582 & .9776 & 9584 & .9583 & .9830 & .9583 & 9586 & 9611 & 9584 \\
\hline .75 & 99121. & 99065 & 99146 & .99125 & .99138 & .9915 & .99153 & .99500 & .99154 & .99154 & .99514 . & 99155 & .99156 & .99184 & 99154 \\
\hline$\overline{\mathrm{m} . \mathrm{e}}$ & 0826 & .0870 & .0562 & .0239 & .0130 & & .0058 & 1549 & 2297 & .2036 & 3283 & .0135 & 2272 & 2150 & .0902 \\
\hline
\end{tabular}

Table 2, $n_{1}=100, n_{2}=100, k_{1}=5, k_{2}=5$ (iid)

\begin{tabular}{|c|c|c|c|c|c|c|c|c|c|c|c|c|c|c|c|}
\hline$p$ & LBonf & LPo & $L C P$ & $L_{E P}$ & $L_{E P}^{G}$ & $\boldsymbol{R}_{\text {sim }}$ & $U_{F K}^{G^{*}}$ & $U_{F K}$ & $U_{C B}$ & $U_{C B}{ }^{G}$ & $U_{Y M}$ & $U J$ & UCP & UPo & UBonf \\
\hline .25 & -5.93 & -1.98 & -7.56 & .0010 & .0049 & .0209 & .0553 & .6307 & 6.369 & 4.288 & .9459 & .7664 & 7.583 & 1.983 & 2881 \\
\hline .3 & -.236 & -.498 & -.143 & .2905 & .3567 & .4343 & .4755 & .8898 & .9550 & .7767 & .9887 & .5735 & .9665 & 1.079 & 6197 \\
\hline .35 & 8062 & .7087 & .8202 & .8238 & .8423 & .8619 & .8670 & .9756 & .8860 & .8795 & .9974 & .8775 & .8941 & 9389 & 8870 \\
\hline .4 & .9738 & 964 & .9757 & .9741 & .9761 & .9776 & .9785 & .9957 & .9792 & .9790 & .9992 & .9791 & .9799 & .9843 & 9792 \\
\hline .45 & 99702 & .99633 & .99721 & 99703 & 99718 & .9973 & .99735 & 99938 & 99737. & 99737. & 99975 & .99738 & 99741 & 99773 & 99737 \\
\hline & 1030 & 1246 & .0998 & 0411 & 0230 & & 0163 & 398 & 051 & 2681 & 3278 & 1804 & 3092 & 3258 & 959 \\
\hline
\end{tabular}

Table 3, $n_{1}=10, n_{2}=100, k_{1}=2, k_{2}=3$ (iid)

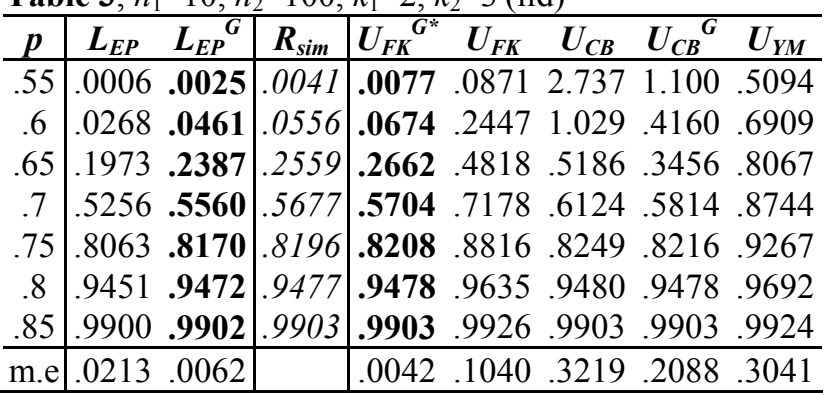

Table 4, (non-iid case)

\begin{tabular}{|c|c|c|c|c|c|c|c|c|}
\hline$n_{1}$ & $n_{2}$ & $k_{1}$ & $\boldsymbol{k}_{2}$ & $\boldsymbol{L}_{E P}$ & $L_{E P}^{G}$ & $\boldsymbol{R}_{\text {sim }}$ & $U_{F K}^{G}$ & \\
\hline 5 & 10 & ( & 5 & & & 606 & & \\
\hline . & & & & & & 5 & 109 & \\
\hline 1 & 1( & 4 & & 6 & . & 393 & 14 & 912 \\
\hline 10 & 5 & 3 & & 1 & .9 & .9969 & 0 & 991 \\
\hline 10 & & 3 & & & & 90 & .7232 & 847 \\
\hline 50 & & 3 & & & & 309 & 2 & 99. \\
\hline 5 & & 2 & & 680 & & .9737 & 41 & 98 \\
\hline & & & & .0241 & 0124 & & $006^{\prime}$ & \\
\hline
\end{tabular}

Table 5, $n_{1}=n_{2}=n, k_{1}=k_{2}=k$, (non-iid case)

\begin{tabular}{|c|c|c|c|c|c|c|c|c|c|c|c|}
\hline$n$ & $k$ & $L P o$ & $L C P$ & $L_{E P}$ & $L_{E P}{ }^{G}$ & $\boldsymbol{R}_{\text {sim }}$ & $U_{F K}^{G^{*}}$ & $\boldsymbol{U}_{F K}$ & UCP & UPo & $\boldsymbol{U J}$ \\
\hline 3 & 2 & .58525 & .73097 & .772476 & .793213 & .8146 & .830545 & .866075 & .91848 & .97235 & .846482 \\
\hline 5 & 2 & -.2992 & -.4607 & .430161 & .478818 & .5324 & .605843 & .715579 & 1.5823 & 1.1806 & .670941 \\
\hline 5 & 3 & .98423 & .98867 & .988126 & .988865 & .9895 & .989852 & .994209 & .99044 & .99204 & .990007 \\
\hline 5 & 4 & .99997 & 99998 & 999980 & 999980 & 9999 & .999980 & 999987 & .99998 & 99999 & .99998 \\
\hline 10 & 2 & -1.235 & -3.724 & .087231 & 121278 & .1676 & 258989 & .434175 & 4.1141 & 1.4210 & .362223 \\
\hline 10 & 3 & .94475 & .96125 & .961058 & .963897 & .9673 & .967980 & .985119 & .97171 & .97743 & .968792 \\
\hline 10 & 4 & .99989 & .99991 & 999907 & 999909 & .9999 & .999911 & 999960 & .99992 & 99992 & 999912 \\
\hline 20 & 2 & -1.490 & -9.708 & .003482 & .007561 & .0167 & .046087 & .156962 & 9.7536 & 1.4976 & 102866 \\
\hline 20 & 3 & .86893 & .90792 & .909099 & .915811 & .9222 & .925656 & .967198 & .93591 & .94939 & .927698 \\
\hline 20 & 4 & .99973 & .99977 & .999762 & 999767 & 9997 & 9999774 & 999906 & .99978 & .99979 & 999774 \\
\hline 50 & 2 & -1.516 & -27.16 & .0000002 & .0000018 & .0000 & .0002575 & .0073614 & 27.166 & 1.5157 & .0023357 \\
\hline 50 & 3 & .66519 & .75990 & .769477 & .785473 & .8022 & .809466 & .915368 & .84044 & .87404 & .814582 \\
\hline 50 & 4 & .99925 & .99935 & 999327 & 999343 & .9993 & .999361 & .999744 & .99937 & .99940 & 999362 \\
\hline 100 & 3 & .39270 & .54786 & .582778 & .608144 & 6372 & .647312 & .835083 & .71599 & .77329 & .655867 \\
\hline 100 & 4 & .99845 & .99865 & .998602 & .998635 & 9986 & .998673 & .999475 & .99868 & .99875 & .998675 \\
\hline m.e. & & .11388 & .06356 & .02307 & .01246 & & .01551 & .06861 & .23491 & .24595 & .03283 \\
\hline
\end{tabular}

